\title{
A Method to Represent Multiple-Output Switching Functions by Using Multi-Valued Decision Diagrams
}

\author{
Tsutomu Sasao
}

Jon T. Butler

\author{
Department of Electrical and \\ Computer Engineering \\ Naval Postgraduate School \\ Monterey, CA 93943-5121, U.S.A.
}

February 19, 1996

\begin{abstract}
Multiple-output switching functions can be simulated by multiple-valued decision diagrams (MDDs) at a significant reduction in computation time. We analyze the following approaches to the representation problem: shared multiple-valued decision diagrams (SMDDs), multi-terminal multiple-valued decision diagrams (MTMDDs), and shared multi-terminal multiple-valued decision diagrams (SMTMDDs). For example, we show that SMDDs tend to be compact, while SMTMDDs tend to be fast. We present an algorithm for grouping input variables and output functions in the MDDs.
\end{abstract}

\section{Introduction}

Various methods exist to represent discrete functions. Among them, graph based representations such as BDDs (binary decision diagrams) are extensively used in logic synthesis, test, and verification [4]. Multiple-valued decision diagrams (MDDs) are multiple-valued extensions of BDDs, and have been used to design logic networks $[14,8,5,9,10]$. Recently, McGeer et al. developed a logic simulator based on MDDs [12]. They showed that the MDD based simulator is orders-of-magnitude faster than a conventional one. Their method is summarized as follows:

1. Represent a given logic function by a BDD.

2. Group $k$ variables into a single $2^{k}$-valued variable forming an MDD from the BDD. Each node in the MDD has $2^{k}$ children. We assume that $n=r k$ is the number of input variables, where $k \geq 2$ is a constant.

3. Translate the MDD into a table on which function evaluation is performed by a sequence of address lookups.

An advantage of the MDD is a reduction in memory accesses needed to evaluate it compared with the BDD from which it was derived. Indeed, grouping $k$ binary inputs together to form a single MDD variable reduces computation time by a factor of $k$. However, there is a tradeoff. Since each group of $k$ binary variables can assume $2^{k}$ possible values, the size of the MDD tends to increase by a factor approaching $2^{k}$. [12] claims that $k=5$ gives the best performance for their prototype simulator. However, they did not show any theoretical or experimental justification.

To represent a logic function efficiently, it is essential to reduce the number of nodes in its MDD. Three methods exist.

1. Group binary variables to form multiple-valued variables.

2. Order the multiple-valued variables in the MDD.

3. Group the outputs.

In this paper, we consider a method of representation for multiple-output functions by MDDs, where $k=2$, i.e., each MDD variable is 4 -valued. Specifically, we consider a method for pairing input variables and pairing output functions to reduce the number of nodes.

We considered the case $k=2$ for the following reason:

1. When $k=2$, a node for a 4 -valued MDD is realized by a "4 to 1 multiplexer," which is available in a CMOS gate array library. Its cost is 4 times that of a 2 -input NAND gate [7]. Also. a node for a 4-valued MDD is realized by a 6input LUT (look-up table) [3, 9]. FPGAs (field programmable gate arrays) with such LUTs are produced by AT\&T [2].

2. $k=2$ is the simplest case, and the design and analysis are easier than for the general case.

Strategies for grouping output functions are also useful for reducing the memory requirement of MTTDDs (Multi-Terminal Ternary Decision Diagrams) that are indispensable in the optimization of AND-EXOR expressions $[8,11]$. Other methods to represent multipleoutput functions using BDDs are developed for fast logic simulation [1]. 


\section{Definitions and Basic Properties}

Definition 2.1 Let $P=\{0,1, \ldots, p-1\}$ and $B=$ $\{0,1\}$. A mapping $f: B^{n} \rightarrow B$ is a switching function. $A$ mapping $f: P^{n} \rightarrow P$ is a p-valued logic function. $A$ mapping $f: P^{n} \rightarrow B$ is a p-valued input two-valued output function.

Definition 2.2 Let $S \subseteq P . X^{S}$ is a literal of $X$, where

$$
X^{S}= \begin{cases}0 & (X \notin S) \\ 1 & (X \in S) .\end{cases}
$$

When $S$ contains only one element, $X^{\{i\}}$ is denoted by $X^{i}$. A product of literals $X_{1}^{S_{1}} X_{2}^{S_{2}} \cdots X_{n}^{S_{n}}$ is a product term that is the $A N D$ of all literals that compose it. The expression

$$
\bigvee_{\left(S_{1}, S_{2}, \ldots, S_{n}\right)} X_{1}^{S_{1}} X_{2}^{S_{2}} \cdots X_{n}^{S_{n}}
$$

is a sum-of-products expression (SOP), where $\bigvee_{\left(S_{1}, S_{2}, \ldots, S_{n}\right)}$ denotes the inclusive-OR of products terms.

Lemma 2.1 An arbitrary p-valued input two-valued output function can be expanded as

$$
\begin{aligned}
f\left(X_{1},\right. & \left.X_{2}, \ldots, X_{r}\right) \\
= & X_{1}^{0} f\left(0, X_{2}, \ldots, X_{r}\right) \vee X_{1}^{1} f\left(1, X_{2}, \ldots, X_{r}\right) \vee \\
& \cdots \vee X_{1}^{p-1} f\left(p-1, X_{2}, \ldots, X_{r}\right) .
\end{aligned}
$$

This is the Shannon expansion with respect to $X_{1}$.

In the derivations that follow, it is convenient to let $x_{i}$ represent a two-valued variable and to let $X_{i}$ represent a $2^{k}$ valued variable corresponding to $k$ twovalued variables. For example, consider a switching function $f\left(x_{1}, x_{2}, \ldots, x_{2 r}\right)$, which can be written as $f\left(X_{1}, X_{2}, \ldots, X_{r}\right)$, where $X_{i}$ is either $0,1,2$, or 3 if $\left(x_{2 i-1}, x_{2 i}\right)=(0,0),(0,1),(1,0)$ and $(1,1)$, respectively. We use the notation $X_{i}=\left(x_{2 i-1}, x_{2 i}\right)$ to denote the relationship between $X_{i}$ and $x_{2 i-1}$ and $x_{2 i}$.

Example 2.1 The switching function shown in Fig. 2.1(a) can be converted into a four-valued input two-valued output function as shown in Fig. 2.1(b), where $X_{1}=\left(x_{1}, x_{2}\right)$ and $X_{2}=\left(x_{3}, x_{4}\right)$.

$$
\text { (End of Example) }
$$

Definition 2.3 Let $f$ be a function. The set of input variables on which $f$ depends is the support of $f$, and is denoted as Support $(f)$.

Example 2.2 Let $f\left(x_{1}, x_{2}, x_{3}, x_{4}\right)=x_{1} x_{2} \vee x_{3} \bar{x}_{4} \vee$ $x_{3} x_{4}$. Then, Support $(f)=\left\{x_{1}, x_{2}, x_{3}\right\}$, since $f$ is also represented as $f=x_{1} x_{2} \vee x_{3}$. (End of Example)

Definition 2.4 A multi-valued decision diagram $(M D D)$ is a generalization of a binary decision diagram (BDD). Specifically, an internal node of an MDD may have more than two children (Fig. 2.2). An $M D D$ having more than two kinds of terminal nodes (e.g., $0,1, \cdots, p-1$ ) is called a multi-terminal $M D D$ (MTMDD).

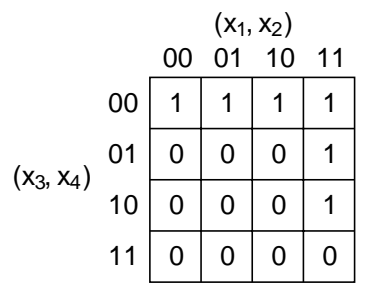

(a) A switching function.

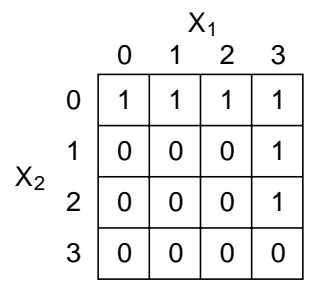

(b) A four-valued input two-valued output function.
Figure 2.1: Example 2.1.

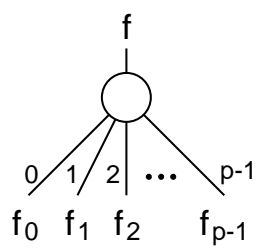

Figure 2.2: Node for an MDD.

For a given BDD, we can group the binary variables, and obtain an MDD.

Example 2.3 Fig. 2.3 shows a BDD for $f=x_{1} \oplus$ $x_{2} \oplus x_{3} \oplus x_{4}$. Let $X_{1}=\left(x_{1}, x_{2}\right)$ and $X_{2}=\left(x_{3}, x_{4}\right)$ represent logic value in the range $\{0,1,2,3\}$. By this, we have a 4-valued input two-valued output function

$$
F\left(X_{1}, X_{2}\right)=X_{1}^{\{0,3\}} X_{2}^{\{1,2\}} \vee X_{1}^{\{1,2\}} X_{2}^{\{0,3\}} .
$$

Fig. 2.4 shows an MDD for F. $\quad$ (End of Example)

\section{Grouping Input Variables}

The number of nodes in an MDD depends on the method of grouping input variables as well as the ordering of the multiple-valued variables.

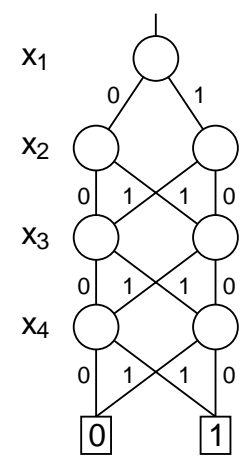

Figure 2.3: $\mathrm{BDD}$ for $f=x_{1} \oplus x_{2} \oplus x_{3} \oplus x_{4}$. 


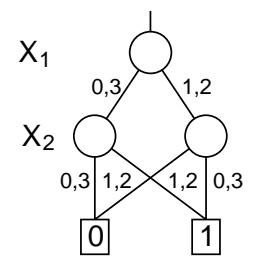

$\mathrm{X}_{1}=\left(\mathrm{x}_{1}, \mathrm{x}_{2}\right)$

$X_{2}=\left(x_{3}, x_{4}\right)$

Figure 2.4: MDD for $X_{1}^{\{0,3\}} X_{2}^{\{1,2\}} \vee X_{1}^{\{1,2\}} X_{2}^{\{0,3\}}$.

Example 3.1 Consider the function $f=x_{1} x_{2}\left(x_{3} \oplus\right.$ $\left.x_{4}\right) \vee \bar{x}_{3} \bar{x}_{4}$.

1) When the input variables are grouped as $X_{1}=$ $\left(x_{1}, x_{2}\right)$ and $X_{2}=\left(x_{3}, x_{4}\right)$, the $M D D$ for $f$ has three non-terminal nodes as shown in Fig. 3.1(a).

2) When the input variables are grouped as $X_{1}=$ $\left(x_{3}, x_{4}\right)$ and $X_{2}=\left(x_{1}, x_{2}\right)$, the $M D D$ for $f$ has only two non-terminal nodes as shown in Fig. 3.1(b).

3) When the input variables are grouped as $X_{1}=$ $\left(x_{1}, x_{3}\right)$ and $X_{2}=\left(x_{2}, x_{4}\right)$, the $M D D$ for $f$ has four non-terminal nodes as shown in Fig. 3.1(c).

Thus, for this function, the grouping of 2) is the best among the three.

(End of Example)

Suppose that the input variables are partitioned into $X_{1}$ and $X_{2}$, where $X_{1}=\left(x_{i}, x_{j}\right)$. By renaming the input variables, we can represent $f$ as $f(X)=f\left(X_{1}, X_{2}\right)$. Next, consider the sub-functions: $f\left(0,0, X_{2}\right), f\left(0,1, X_{2}\right), f\left(1,0, X_{2}\right)$, and $f\left(1,1, X_{2}\right)$. Let $\mu$ be the number of distinct non-constant functions among these four functions. Let the BDD representing $f$ have $x_{i}$ as the highest variable and $x_{j}$ as the second highest. Since $f$ depends on $x_{i}$, the root node representing $f$ has two children nodes representing $f_{x_{i}=0}$ and $f_{x_{i}=1}$. Since there are $\mu$ nonconstant subfunctions associated with assignments of values to $x_{i}$ and $x_{j}$, the number of non-terminal nodes in the lower level is $\mu$. As an example, consider $f\left(x_{1}, x_{2}, x_{3}, x_{4}\right)=x_{1} \oplus x_{2} \oplus x_{3} \oplus x_{4}$, and let $\left(x_{1}, x_{2}\right)=$ $\left(x_{i}, x_{j}\right)$. Among the four subfunctions, $f\left(0,0, x_{3}, x_{4}\right)$, $f\left(0,1, x_{3}, x_{4}\right), f\left(1,0, x_{3}, x_{4}\right)$, and $f\left(1,1, x_{3}, x_{4}\right)$, are two distinct ones, $x_{3} \oplus x_{4}$ and $x_{3} \oplus x_{4} \oplus 1$. Thus, $\mu=2$, and there are two non-terminal nodes at the second level. This is shown in Fig. 2.3. Further, in the multiple-valued version of a function $f$, represented as $f\left(X_{1}, X_{2}, \ldots, X_{r}\right)$, where $X_{1}=\left(x_{i}, x_{j}\right)$, there will also be $\mu$ nodes. In our running example, there are two nodes at the $X_{2}$ level in Fig. 2.4, which is an MDD representation of the BDD in Fig. 2.3. This shows that a grouping in the BDD that reduces nodes tends to reduce nodes in the equivalent MDD. If $\mu \leq 3$. then $\left(x_{i}, x_{j}\right)$ is a candidate pair. It is clear that if $f$ is partially symmetric with respect to $x_{i}$ and $x_{j}$, then $\mu \leq 3$.

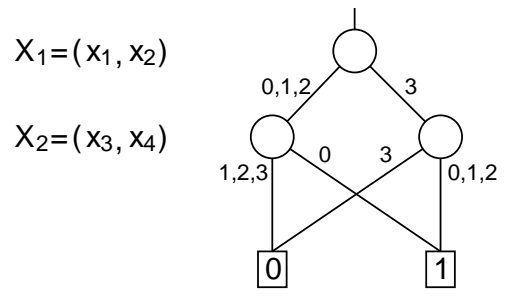

(a)

$X_{1}=\left(x_{3}, x_{4}\right)$

$X_{2}=\left(x_{1}, x_{2}\right)$

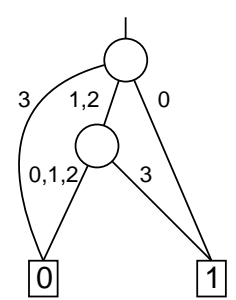

(b)

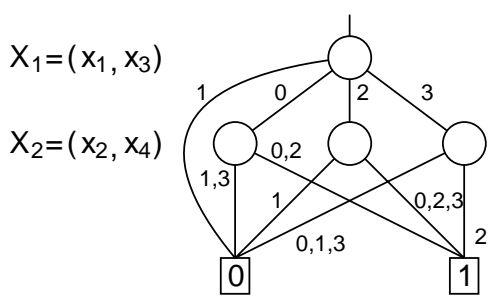

(c)

Figure 3.1: MDD for $f=x_{1} x_{2}\left(x_{3} \oplus x_{4}\right) \vee \bar{x}_{3} \bar{x}_{4}$.

\section{Representation of Multiple-Output} Functions

Practical logic networks have, usually, many outputs. In this section, we consider various methods to represent multiple-output functions by MDDs.

\subsection{Shared MDD}

Fig. 4.1 shows the general structure of a shared MDD (SMDD), where each function $f_{i}(i=$ $0,1, \ldots, m-1$ ) has its corresponding MDD. These MDDs may share sub-graphs. Above the root nodes, the output selection variables are used. The advantage of this data structure for use in a simulator is that the sizes of MDDs are relatively small. The disadvantage is that we need to evaluate MDDs $m$ times. This is because the MDD produces only one output for each assignment of values to the output selection variables. Its use in a simulation package requires the production of all outputs sequentially, and this results in a slow response time.

Example 4.1 Fig. 4.2 shows the shared BDD $(S B D D)$ for the two-input two-output function in Table 4.1.

(End of Example)

\subsection{Multi-Terminal MDD}

Fig. 4.3 shows the general structure of a multiterminal MDD (MTMDD), where the terminals are 


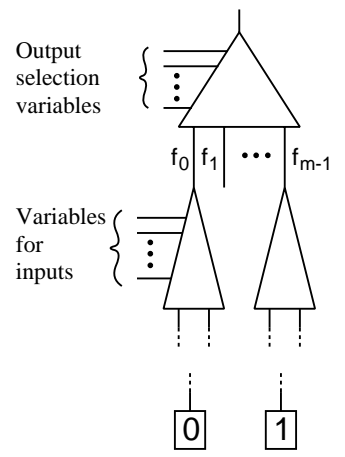

Figure 4.1: General structure of an SMDD.

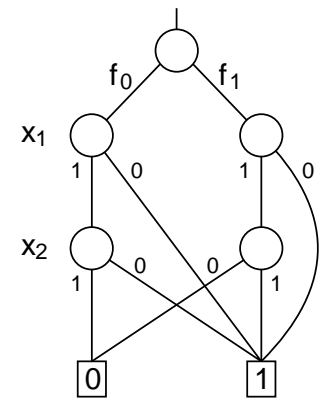

Figure 4.2: SBDD for 2-input 2-output function.

$m$-bit binary vectors. In a conventional MDD, an assignment of values to the variables corresponds to a path that terminates on a single binary value. This value is the function value for that assignment. However, in an MTMDD, a path terminates on a vector of $m$-bits corresponding to $m$ functions. The merit of this data structure is that the values of all the outputs can be evaluated simultaneously. This is because the MDD produces all the output values for each assignment of values to the input variables. The demerit is that the size of the MTMDDs tends to be larger than that of SMDDs, when $m$ is large.

Example 4.2 Fig. 4.4 shows an MTBDD for the two-input two-output function in Table 4.1.

(End of Example)

The above example shows a case where an MTBDD represents functions efficiently. However, the next ex-

Table 4.1:

\begin{tabular}{cc|cc}
\hline \multicolumn{2}{c|}{ Input } & \multicolumn{2}{c}{ Output } \\
\hline$x_{1}$ & $x_{2}$ & $f_{0}$ & $f_{1}$ \\
\hline 0 & 0 & 1 & 1 \\
0 & 1 & 1 & 1 \\
1 & 0 & 1 & 0 \\
1 & 1 & 0 & 1 \\
\hline
\end{tabular}

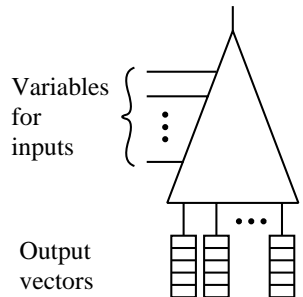

Figure 4.3: General structure of an MTMDD.

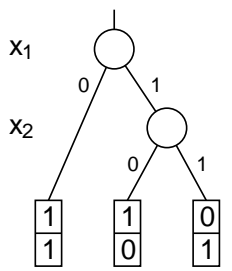

Figure 4.4: MTBDD for 2-input 2-output function. ample shows a case where an MTBDD is quite inefficient.

Example 4.3 Consider the three-output function: $f_{0}=x_{1}, f_{1}=x_{2}$, and $f_{2}=x_{3}$. As shown in Fig. 4.5, the $S B D D$ is simple, but the $M T B D D$ is the complete tree for three variables.

(End of Example)

The above example suggests that a conversion from an SBDD to an MTBDD may increase the size of the structure exponentially. Note that, on the other hand, a conversion from an MTBDD to an SBDD can increase nodes by at most $m$ times, where $m$ is the number of outputs. Experimental results show that, in many cases, when $m$ is large, the size of an MTBDD is larger than the size of the equivalent SBDD. The number of nodes tends to be larger than the equivalent SMDD.

\subsection{MDD with Output Selection Vari- ables}

Fig. 4.6 shows the general structure of an MDD with output selection variables. This data structure is the same as the SMDD, except that the output selection variables are interchanged with the input variables. Also, in this case, we need additional time to select the output functions. However, the time to evaluate $m$ outputs can be less than that of the equivalent $\mathrm{SMDD}$, if we use a special technique in a simulation program.

\subsection{Shared MTMDD}

Fig. 4.7 shows the general structure of a shared MTMDD (SMTMDD). This data structure is a combination of an SMDD and an MTMDD. In an SMTMDD, the output selection variables select a set of outputs (in this example, two), and each MDD for $g_{i}$ $(i=0,1, \ldots, m / 2)$ represents the set of outputs. Its 


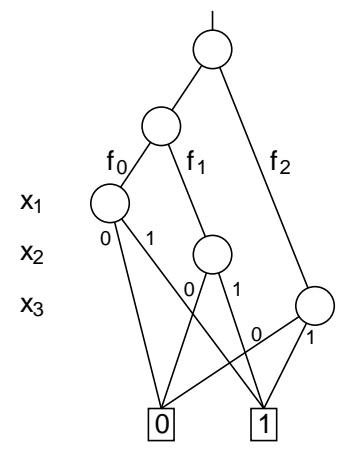

(a) SBDD

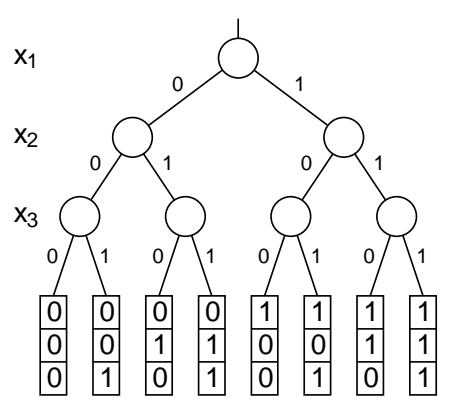

(b) MTBDD

Figure 4.5: SBDD and MTBDD.

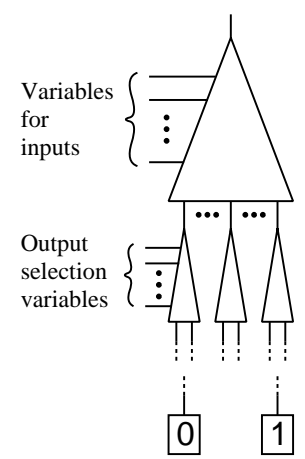

Figure 4.6: General structure of an MDD with output selection variable.

merit is that several (in this example, two) functions are evaluated simultaneously. Also, the functions can be grouped so that the size of the resulting MDD remains moderate.

\subsection{Strategies for Grouping Output Func- tions}

Consider an MTMDD for two-output function $\left(f_{i}, f_{j}\right)$. In general, the MTMDD has $(0,0),(0,1)$, $(1,0)$, and $(1,1)$ as terminal nodes. However if $f_{i} f_{j}=$ 0 , then $(1,1)$ never appears as a terminal node in the MTMDD for $\left(f_{i}, f_{j}\right)$. Thus, this grouping of the out-

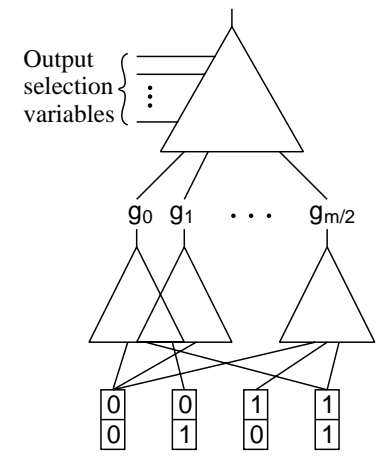

Figure 4.7: General structure of an SMTMDD.

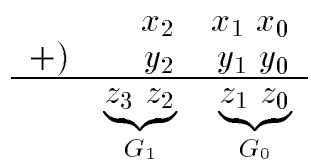

Figure 4.8: Three-bit adder (adr3).

put functions tends to produce a smaller MDD, since the number of terminal nodes is at most three. Similarly, if $f_{i} \bar{f}_{j}=0, \bar{f}_{i} f_{j}=0$, or $\bar{f}_{i} \bar{f}_{j}=0$, then $f_{i}$ and $f_{j}$ is also a candidate pair. On the contrary, if $f_{i}$ and $f_{j}$ have disjoint supports, then they should not be paired. but represented by separate MDDs.

Example 4.4 Consider the three-bit adder (adr3) shown in Fig. 4.8. Assume that the input variables and output functions are paired as follows: $X_{1}=\left(x_{2}, y_{2}\right)$, $X_{2}=\left(x_{1}, y_{1}\right), X_{3}=\left(x_{0}, y_{0}\right), G_{1}=\left(z_{3}, z_{2}\right), G_{0}=$ $\left(z_{1}, z_{0}\right)$. Note that

$$
\begin{aligned}
& \text { Support }\left(z_{0}\right)=\left\{x_{0}, y_{0}\right\}, \\
& \operatorname{Support}\left(z_{1}\right)=\left\{x_{0}, y_{0}, x_{1}, y_{1}\right\}, \\
& \operatorname{Support}\left(z_{2}\right)=\left\{x_{0}, y_{0}, x_{1}, y_{1}, x_{2}, y_{2}\right\}, \text { and } \\
& \operatorname{Support}\left(z_{3}\right)=\left\{x_{0}, y_{0}, x_{1}, y_{1}, x_{2}, y_{2}\right\} .
\end{aligned}
$$

Fig. 4.9 shows the SMDD: Four MDDs for $z_{3}, z_{2}, z_{1}$, and $z_{0}$ are realized. In this case, the number of nodes is small. However, the simulation is slow since we have to evaluate MDDs four times, once for each of the four output functions. Fig. 4.10 shows the MT$M D D$ : A single $M D D$ with 4-bit terminal nodes. In this case, the number of nodes is large. However, the simulation is fast, since four outputs are evaluated simultaneously. Fig. 4.11 shows the SMTMDD, a pair of MDDs with 2-bit terminal nodes. In this case, the number of nodes is moderate. The simulation time is one-half that of the SMDD, since two outputs are evaluated simultaneously.

(End of Example)

\section{Optimization Algorithm for MDDs}

For simplicity, we assume that $n=2 r$, and $m$ is an even number, where $n$ denotes the number of in- 


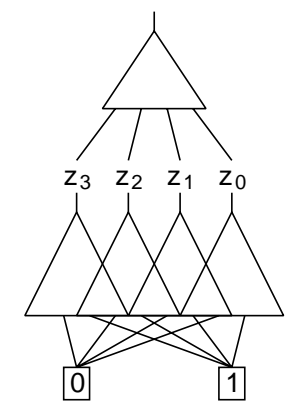

Figure 4.9: SMDD.
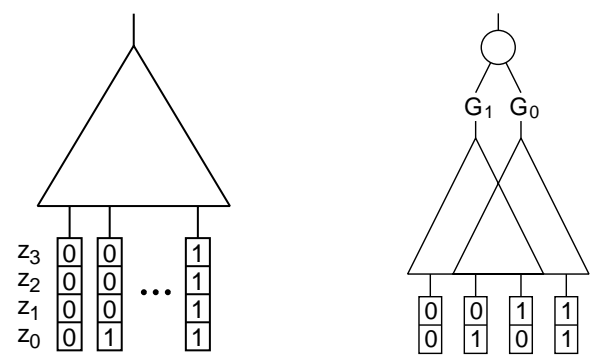

Figure 4.10: MTMDD. Figure 4.11: SMTMDD.

put variables, and $m$ denotes the number of output functions.

\subsection{Grouping and Ordering of Input Vari- ables}

Algorithm 5.1 (Group the Input Variables)

1. Construct an $S B D D$ from the given specification.

2. Find a good initial ordering of the input variables for the BDD, by interchanging adjacent variables.

3. Count the nodes in the MDD. This is done in a $B D D$ data structure: If the $B D D$ contains disjoint subgraphs, Fig. 5.1(a), (b), or (c), then the numbers of the corresponding nodes in the $M D D$ are counted as one, two, or three, respectively. In Fig. 5.1(a), three BDD nodes are replaced by one MDD node. However, in Fig. 5.1(b), the $B D D$ nodes are replaced by two $M D D$ nodes. In Fig. 5.1(c), we need three MDD nodes. Thus, the number of nodes in an $M D D$ is more than one third of that for the corresponding $B D D$.

Algorithm 5.2 (Order Input Variables in the MDD)

1. Construct an SMDD by Algorithm 5.1.

2. Reduce the number of nodes in the MDD by interchanging the adjacent variables.

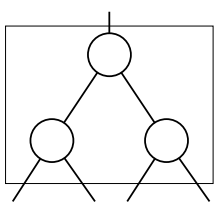

(a)

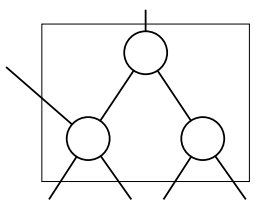

(b)

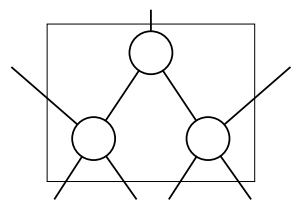

(c)
Figure 5.1: Enumeration of MDD nodes.

Table 6.1: Number of MDD nodes of represent adder.

\begin{tabular}{|l|r|r|r|r|r|r|}
\hline Name & in & out & SBDD & SMDD & MTMDD & SMTMDD \\
\hline adr3 & 6 & 4 & 25 & 11 & 26 & 15 \\
\hline adr5 & 10 & 6 & 46 & 18 & 120 & 26 \\
\hline adr7 & 14 & 8 & 65 & 25 & 502 & 37 \\
\hline $\operatorname{adr} 9$ & 18 & 10 & 87 & 31 & 2036 & 49 \\
\hline
\end{tabular}

\subsection{Grouping of Output Functions}

Algorithm 5.3 (Group the Output Functions)

Let $f_{0}, f_{1}, \ldots, f_{m-1}$ be the output functions. Let $m$ be even.

1. Construct an $S B D D$ from the given specification.

2. For $(0 \leq i \leq m-1)$, construct the BDD representing $f_{i}$. Calculate $w_{i}$, the number of nodes.

3. For $(0 \leq i<j \leq m-1)$, construct an $S B D D$ representing $f_{i}$ and $f_{j}$. Count $w_{i j}$, the number of nodes.

4. Let $W_{i j} \leftarrow w_{i}+w_{j}-w_{i j}$. Note that $W_{i j}$ is the reduction in nodes achived by pairing $f_{i}$ and $f_{j}$.

5. Consider the complete graph $G$ with $m$ nodes whose edges have weight $W_{i j}$. Obtain the maximum matching for $G$. That is, choose a set $E$ of $m / 2$ edges such that each node is incident to exactly one edge, and $\sum_{i<j} W_{i j}$ is maximum, where the sum is over all possible edges in $E$. We use a branch and bound algorithm.

6. Group the output functions according to the maximum matching of $G$.

\section{Experimental Results}

[12] did not show the number of MDD nodes, so the comparison with their method is not made.

\subsection{Representation of Adders}

$n$-bit adders $(\operatorname{adr} n)$ for $n=3,5,7$, and 9 are represented by SBDDs (shared BDDs), SMDDs, MTMDDs, and SMTMDDs. Table 6.1 compares the number of nodes. Note that the number of terminal nodes for SBDDs, SMDDs, MTMDDs, and SMTMDDs are 2, $2,2^{n+1}-1$, and 4 , respectively. SMDDs require the fewest nodes among the three types of MDDs, and MTMDDs require the most number of nodes. The number of nodes in SMTMDDs is not so large, but the simulation time is half that of SMDDs, since two functions are evaluated simultaneously. 
Table 6.2: Number of MDD nodes of represent various functions.

\begin{tabular}{|l|r|r|r|r|r|r|}
\hline Name & in & out & SBDD & SMDD & MTMDD & SMTMDD \\
\hline alu2 & 10 & 8 & 82 & 44 & 174 & 63 \\
apla & 10 & 12 & 123 & 67 & 85 & 90 \\
bc0 & 26 & 11 & 624 & 363 & 209 & 378 \\
clip & 10 & 5 & 138 & 56 & 95 & 91 \\
dc2 & 8 & 7 & 74 & 41 & 136 & 41 \\
dk17 & 10 & 11 & 82 & 44 & 54 & 67 \\
dk27 & 9 & 9 & 37 & 22 & 39 & 25 \\
f51m & 8 & 8 & 83 & 43 & 341 & 51 \\
in1 & 16 & 17 & 580 & 322 & 188 & 437 \\
in2 & 19 & 10 & 301 & 164 & 189 & 226 \\
inc & 7 & 9 & 89 & 46 & 44 & 48 \\
misex1 & 8 & 7 & 45 & 26 & 19 & 33 \\
misex3 & 14 & 14 & 580 & 322 & 1773 & 351 \\
misj & 35 & 14 & 59 & 35 & 3492 & 54 \\
mlp4 & 8 & 8 & 151 & 79 & 170 & 92 \\
radd & 8 & 5 & 35 & 15 & 57 & 21 \\
rd53 & 5 & 3 & 27 & 14 & 15 & 15 \\
rd73 & 7 & 3 & 47 & 24 & 24 & 24 \\
rd84 & 8 & 4 & 64 & 33 & 25 & 31 \\
rot8 & 8 & 5 & 85 & 42 & 47 & 39 \\
sao2 & 10 & 4 & 99 & 49 & 36 & 52 \\
sex & 9 & 14 & 63 & 40 & 105 & 50 \\
sqr8 & 8 & 16 & 272 & 132 & 341 & 150 \\
tial & 14 & 8 & 774 & 380 & 388 & 624 \\
ts10 & 22 & 16 & 177 & 73 & 240297 & 79 \\
x6dn & 39 & 5 & 263 & 149 & 148 & 188 \\
z5xp1 & 5 & 10 & 82 & 41 & 213 & 55 \\
\hline & & & & & & \\
\hline
\end{tabular}

\subsection{Representation of Other Functions}

Other benchmark functions are represented by SBDDs, SMDDs, MTMDDs, and SMTMDDs. Table 6.2 compares the number of nodes. Each MDD (BDD) was optimized by a heuristic program independently. Thus, MDDs (BDDs) for the same function may use different ordering of the input variables. In many cases, SMDDs required the fewest nodes among three MDDs. MTMDDs sometimes required excessive number of nodes, although they sometimes require the fewest nodes. SMTMDDs were not as large as MTMDDs, but were usually larger than SMDDs.

\section{Conclusion and Comments}

In this paper, we considered various methods to represent multiple-output functions by using MDDs. We presented algorithms for grouping input variables and output functions to reduce the number of nodes in MDDs. These methods are useful for fast logic simulation. The technique in 5.2 can be also used as a BDD data structure for representing multiple-output logic functions.

\section{Acknowledgments}

This work was supported in part by a Grant in Aid for Scientific Research of the Ministry of Education, Science and Culture of Japan, and by a JSPS Fellowship. Mr. M. Matsuura developed the program and did the experiments.

\section{References}

[1] P. Ashar and S. Malik, "Fast functional simulation using branching programs," in Proc. of the International Conference on Computer-Aided Design, Nov. 1995.

[2] AT\&T, ATET ORCA (Optimized Reconfigurable Cell Array) Series FPGA, Product Brief, April. 1993.

[3] S. D. Brown, R. J. Francis, J. Rose, and Z. G. Vranesic, Field Programmable Gate Arrays, Kluwer Academic Publishers, Boston 1992.

[4] R. E. Bryant, "Graph-based algorithms for Boolean function manipulation," IEEE Trans. Comput. Vol. C-35, No. 8, Aug. 1986, pp. 677691.

[5] D. M. Miller, "Multiple-valued logic design tools," Proc. of International Symposium on Multiple Valued Logic, May 1993, pp. 2-11.

[6] S. Minato, "Graph-based representation of discrete functions," in T. Sasao and M. Fujita, (e.d.) Representations of Discrete Functions, Kluwer Academic Publishers, 1996 (to be published).

[7] NEC Corporation, CMOS-8 Family Ver. 3.0 Block Libaray, User's Manual, 1994.

[8] T. Sasao (ed.), Logic Synthesis and Optimization, Kluwer Academic Publishers (1993-01).

[9] T. Sasao and J. T. Butler, "A design method for look-up table type FPGA by pseudo-Kronecker expansion," Proc. of International Symposium on Multiple Valued Logic, Boston, MA, May 25-27, 1994, pp. 97-106.

[10] T. Sasao and J. T. Butler, "Planar multiplevalued decision diagrams," Proc. of International Symposium on Multiple Valued Logic, Bloomington, Indiana, May 23-25, 1995, pp. 28-35.

[11] T. Sasao and F. Izuhara, "Exact minimization of fixed polarity Reed-Muller expressions using multi-terminal EXOR ternary decision diagram," in T. Sasao and M. Fujita, (e.d.) Representations of Discrete Functions, Kluwer Academic Publishers, 1996 (to be published).

[12] P. C. McGeer, K. L. McMillan, A. Saldanha, A. L. Sangiovanni-Vincentelli, P. Scaglia, "Fast discrete function evaluation using decision diagrams," International Workshop on Logic Synthesis, Lake Thahoe, May, 1995, pp. 6_1-6_9. Also, in Proc. of the International Conference on Computer-Aided Design, pp. 402-407, Nov. 1995.

[13] M. Davio, J-P. Deschamps, and A. Thayse, Discrete and Switching Functions, McGraw-Hill International, 1978.

[14] A. Srinivasan, T. Kam. S. Malik, and R. K. Brayton, "Algorithm for discrete functions manipulation," in Proc. ICCAD-90, pp. 92-95, Nov. 11-15, 1990, Stanta Clara, CA. 